\title{
Catheter-Associated Urinary Tract Infections in Adults: Diagnosis, Treatment, and Prevention
}

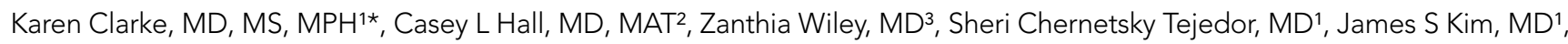
Lisa Reif, MSN, RN, APRN-CCNS, CCRN4, Lucy Witt, MD¹, Jesse T Jacob, MD³

${ }^{1}$ Division of Hospital Medicine, Department of Medicine, Emory University School of Medicine, Atlanta, Georgia; ${ }^{2}$ Division of Neuroscience Critical Care, Departments of Neurology and Neurosurgery, Emory University School of Medicine, Atlanta, Georgia; ${ }^{3}$ Division of Infectious Diseases, Department of Medicine, Emory University School of Medicine, Atlanta, Georgia; ${ }^{4}$ Emory Healthcare, Atlanta, Georgia.

Urinary tract infections (UTIs) are among the most common healthcare-associated infections, and $70 \%-80 \%$ are catheter-associated urinary tract infections (CAUTIs). About $25 \%$ of hospitalized patients have an indwelling urinary catheter placed during their hospital stay, and therefore, are at risk for CAUTIs, which have been associated with worse patient outcomes. Additionally, hospitals face a significant financial impact since the
Centers for Medicare \& Medicaid Services incentive program penalizes hospitals with higher than expected CAUTIs. Hospitalists care for many patients with indwelling urinary catheters and should be aware of and engage in processes that reduce the rate of CAUTIs. This article will discuss the diagnosis, treatment, and prevention of CAUTIs in adults. Journal of Hospital Medicine 2020;15:552-556. (C) 2020 Society of Hospital Medicine


very day in the United States, approximately $4 \%$ of patients in acute care hospitals have at least one hospital-acquired infection (HAI). ${ }^{1,2}$ Among the top 10 causes of death in the United States, HAls are associated with increased morbidity, mortality, and hospital length of stay (LOS). ${ }^{2}$ The direct medical cost of treating HAls is substantial for both hospitals and patients.,.$^{3,4}$ Urinary tract infections (UTIs) are a leading cause of $\mathrm{HAl}$, and $70 \%-80 \%$ of these are catheter-associated urinary tract infections (CAUTIs). 5,6 In 2016, 26,983 CAUTIs occurred in acute care hospitals. ${ }^{7}$ The high incidence of CAUTI can substantially contribute to morbidity, length of stay, and mortality. . $^{-11}$

The recognition that a substantial proportion of HAls may be preventable, including $55 \%-70 \%$ of CAUTIs, ${ }^{12}$ has resulted in implementing multiple strategies to reduce CAUTI rates. ${ }^{13-17}$ These include simple prevention interventions such as avoiding placement of unnecessary indwelling urinary catheters and early removal of urinary catheters when they are no longer clinically indicated. Hospitalists are responsible for the care of many, if not most, inpatients with indwelling urinary catheters and are integral in antimicrobial stewardship efforts surrounding CAUTls. ${ }^{18}$ Diagnostic stewardship, including appropriate urine specimen ordering, collection, processing, and reporting, works synergistically with antimicrobial stewardship and allows for appropriate antibiotic prescribing in symptomatic patients. ${ }^{19}$

*Corresponding Author: Karen Clarke, MD; Email: karen.clarke@ emoryhealthcare.org; Telephone: 678-857-9924.

Published online first September 18, 2019.

Received: December 6, 2018; Revised: July 1, 2019; Accepted: July 17, 2019

๑) 2019 Society of Hospital Medicine DOI 10.12788/jhm.3292

\section{DEFINITIONS}

CAUTIs can be defined using either clinical or surveillance definitions. Clinical definitions are used at the bedside and take individual clinical characteristics into consideration, but vary among clinicians since there is no gold standard. Abnormal laboratory urinary findings in the absence of symptoms are not sufficient for the diagnosis of UTI, including CAUTI. Surveillance definitions, such as those used by the Centers for Disease Control and Prevention, ${ }^{20}$ are designed to be simple, easily applicable in any healthcare setting, and standardized to all patients. Surveillance definitions generally include at least one systemic or local symptom (such as fever or dysuria) and positive urine culture in a patient with an indwelling urinary catheter (or within 48 hours after its removal).

Pyuria is leukocytes or white blood cells (WBCs) in a urine specimen, with a threshold of $>10$ WBCs/high-power field using urine microscopy. The predictive value of different thresholds of pyuria for UTI is unclear.

Bacteriuria denotes the presence (on microscopy or culture) of bacteria in the urine. In a patient without signs or symptoms of a UTI, this is termed asymptomatic bacteriuria (ASB). A full discussion of bacteriuria, a major reason for inappropriate antibiotic use, is beyond the scope of this article but is discussed in a recent guideline. ${ }^{21}$

Urinary tract infections are usually characterized by a clinical syndrome along with evidence of pyuria and/or bacteriuria. The two major clinical syndromes observed are lower UTI (cystitis or bladder infections) and upper UTI (pyelonephritis or kidney infections). Rarely, patients may develop asymptomatic bacteremic UTI, in which blood and urine cultures grow the same pathogen in the absence of clinical symptoms. Table 1 summarizes the key points for these definitions. 
Pyuria is the presence of white blood cells in a urine specimen. Greater than 10 WBCs per high-power field using urine microscopy is generally considered significant.

Asymptomatic bacteriuria denotes the presence (on microscopy or culture) of bacteria in a urine sample from a patient without signs or symptoms of a UTI.

UTIs are characterized by the presence of a clinical syndrome supported by evidence of pyuria and/or bacteriuria. The two major clinical syndromes are lower UTI (cystitis) or upper UTI (pyelonephritis).

CAUTI develop in the setting of a urinary catheter. Such patients may have symptomatic bacteriuria (UTI) or rarely asymptomatic bacteremic UTI.

Abbreviations: CAUTI, catheter-associated urinary tract infection; UTI, urinary tract infection.

\section{CAUSES AND RISK FACTORS FOR CAUTI}

Bacterial biofilm can form on the inner and outer surfaces of an indwelling urinary catheter following its insertion and can be associated with bacteriuria and CAUTI. ${ }^{22,23}$ The biofilm comprises bacteria from the periurethral area that migrate upwards from a colonized drainage system. Bacteria present in the biofilm tend to exhibit slow growth, are protected from antibiotic exposure, and have less susceptibility to these agents. ${ }^{22-24}$ When a mature biofilm has formed, catheter removal may be necessary for source control and to facilitate effective antimicrobial treatment. The pathogens that most commonly cause CAUTIs are Escherichia coli (23.9\%), Pseudomonas aeruginosa (10.3\%), and Klebsiella pneumoniae/ oxytoca (10.1\%). ${ }^{25}$ Although urine cultures often grow yeast, particularly Candida spp, nonbacterial pathogens rarely cause UTI.

The risk of developing a CAUTI is directly related to catheter dwell time. ${ }^{26,27}$ For catheterized patients, the rate of development of catheter-associated bacteriuria is approximately $3 \%$ to $7 \%$ per day ${ }^{14,28}$ and is more common in the elderly and females. The likelihood of bacteriuria approaches $100 \%$ if a patient has an indwelling urinary catheter for $\geq 30$ days, ${ }^{27,29,30}$ which is part of the rationale for why a urine culture alone is not sufficient to diagnose a CAUTI. While bacteriuria is a risk factor for UTI, the frequency of progression from bacteriuria to CAUTI is low and treating ASB does not decrease the risk of future CAUTI. Other risk factors for the development of CAUTI include urinary tract instrumentation, diabetes mellitus, and malnutrition. ${ }^{31,32}$

The two most important factors that lead to the development of CAUTIs and have been the main focus of quality improvement areas are unnecessary urinary catheter placement and inappropriate delay in removing a catheter when it is no longer needed. ${ }^{26,33}$ Unfortunately, 38\% of attending physicians are unaware that their patients have a urinary catheter in place. ${ }^{34}$ Furthermore, in $20 \%$ to $50 \%$ of cases, there is no clear indication for catheter placement. ${ }^{2,34}$

\section{DIAGNOSIS OF CAUTI}

A CAUTI diagnosis is typically one of exclusion, as most patients present with fever and no apparent alternative source. ${ }^{14,29}$ Since catheterized patients may not exhibit common cystitis symptoms, ${ }^{29}$ most who develop CAUTI present with fever alone. However, most fevers in patients with bacteriuria and catheters are not CAUTIs and can be attributed to other sources. If a patient with an indwelling urinary catheter develops a fever and there is a suspicion of a CAUTI, careful evaluation is warranted for alternative sources of infection. This particularly applies to patients with severe systemic illness, such as hypotension or systemic inflammatory response syndrome, since these are unusual manifestations of CAUTI. The presence of either cloudy or malodorous urine does not indicate a UTI, and should not be the sole rationale for obtaining a urine culture.

Diagnostic workup of fever should include a clinical assessment of the patient. Indeed, professional guidelines recommend against obtaining a urine culture routinely for fever, unless invasive UTI risk is elevated, such as in patients with neutropenia, history of renal transplantation, or recent genitourinary surgery. ${ }^{35}$ Diagnostic stewardship, focusing on the appropriate use of urine cultures, can reduce CAUTI rates. ${ }^{36}$ For catheterized patients, hospitals are increasingly adopting reflex urine culture, where urine is simultaneously collected for a urinalysis and urine culture, but a urine culture is performed only if the urinalysis is positive for a predetermined threshold for pyuria, leukocyte esterase, or both. However, the use of reflex urine cultures remains an area of debate. ${ }^{37}$ In addition, the Infectious Diseases Society of America recommends against screening for or treating ASB in patients with either short-term ( $<30$ days) or long-term indwelling urethral catheters. ${ }^{21}$

Ideally, a urine culture should be obtained by collecting a midstream sample. In catheterized patients, a sample should be obtained after removal of the catheter; or, in patients with a clinical indication for ongoing catheterization, a sample should be obtained after a new catheter has been placed. ${ }^{14}$ If an indwelling urinary catheter must be continued, the recommendation is to disinfect the drainage system's aspiration port and then obtain a urine culture. Urine should never be obtained from a catheter collection bag. Table 2 summarizes best practices for diagnosis of CAUTI.

\section{TREATMENT OF CAUTI}

For all CAUTIs, an indwelling urinary catheter should be removed as soon as possible. If an indwelling urinary catheter remains necessary, but the existing catheter has been in place longer than two weeks, a new catheter should be placed before initiating antibiotic therapy ${ }^{14}$ to accelerate symptom resolution and reduce the likelihood of relapse or recurrence. ${ }^{32}$

Urinary tract agents such as fosfomycin and nitrofurantoin are recommended as first-line agents for simple cystitis in women and can be used in patients with lower UTI and sufficient renal function to achieve adequate drug concentration in urine. Upper UTIs require antibiotics with good penetration into renal parenchyma such as ceftriaxone. If empiric antimicrobial thera- 
TABLE 2. Best Practices for Diagnosis and Treatment of CAUTI

DIAGNOSIS

1. Most patients with a CAUTI present with fever with no apparent alternative source; however, most fevers in patients with bacteriuria and catheters are attributable to sources other than urine.

2. All fever workups should include a clinical assessment of the patient and appropriate targeting of the diagnostic workup to the most likely source. Urine cultures should be obtained when UTI is suspected, not routinely for any fever.

3. The best way to obtain a specimen for a urine culture is by first removing an unnecessary indwelling urinary catheter, and then obtaining a midstream sample.

4. Ideally, the indwelling urinary catheter should be removed altogether. If it is still indicated, then it should be replaced before collecting the urine specimen for culture to minimize detection of colonization.

\section{TREATMENT}

1. Ideally, antimicrobial therapy should be selected based on urine culture results.

2. If antimicrobial therapy must be started empirically, then review the patient's prior urine culture results, the local antibiogram, and/or a clinical practice guideline to choose appropriate initial therapy.

3. The optimal duration of antimicrobial therapy for a CAUTI is unclear, but most patients can be treated with a relatively short duration of therapy ( $\leq 7$ days).

Abbreviations: CAUTI, catheter-associated urinary tract infection; UTI, urinary tract infection.

py is needed before culture results are available, then previous urine culture results, local antibiograms, or practice guidelines can guide selection. Definitive antimicrobial therapy should be based on urine culture results. It is important to narrow empiric therapy ${ }^{14}$ to reduce risk of Clostridioides difficile infection and emergence of other resistant bacteria. Fluoroquinolones should be avoided for lower UTIs because of these risks and multiple United States Food and Drug Administration warnings. ${ }^{38-40}$

The optimal duration of antimicrobial therapy for a CAU$\mathrm{TI}$ is unclear; ${ }^{14}$ however, most patients can be treated with a relatively short duration of therapy ( $\leq 7$ days) if they respond promptly to therapy. Patients with a slow response to therapy may require 10-14 days of treatment. ${ }^{14}$ Table 2 summarizes best practices for the treatment of CAUTI.

\section{STRATEGIES FOR CAUTI PREVENTION}

Since CAUTI is predicated on the presence of an indwelling urinary catheter, the simplest way to reduce CAUTI is to avoid placing or retaining unnecessary catheters. Some examples of appropriate indications ${ }^{9}$ for placement and maintenance of an indwelling urinary catheter are listed below:

1. Accurate measurement of urinary output in severely ill patients;

2. Improved comfort for patients receiving end-of-life care;

3. Acute urinary retention or bladder outlet obstruction;

4. Need for a period of prolonged immobilization (eg, potentially unstable lumbar or thoracic spine, or has multiple traumatic injuries);

5. Selected surgical procedures, such as urologic procedures and those that are expected to have a prolonged duration, require intraoperative monitoring of urine output, require the administration of either large volumes of intravenous infusions or diuretics;

6. To promote healing of open perineal or sacral wounds in patients with incontinence;

7. Neurogenic bladder; and

8. Hematuria with clots
To increase the timely removal of urinary catheters that are no longer indicated, daily assessment of catheter necessity must be an integral part of clinicians' workflow. ${ }^{32,33}$ Alternatives, such as external catheters or intermittent catheterization, should be considered before indwelling urinary catheter placement since both options are associated with a reduced CAUTI risk. ${ }^{41-44}$ Although indwelling urinary catheters can be seen as being more convenient for both patients and healthcare providers, many patients have expressed a preference for the use of intermittent catheterization compared with indwelling urinary catheterization. ${ }^{43}$

For urinary retention, bladder scanning can noninvasively assess the amount of residual urine in a patient's bladder and can avoid unnecessary insertion of an indwelling urinary catheter. However, if indwelling urinary catheters are ultimately needed, they must be inserted and maintained appropriately. Of note, the use of antibiotic-impregnated catheters has not been shown to reduce CAUTI rates significantly. ${ }^{45}$

CAUTI prevention requires a multidisciplinary collaborative approach. Nurse-driven protocols and checklists to remove indwelling urinary catheters that are no longer indicated can be very effective. ${ }^{46,47}$ Automatic stop orders and catheter removal reminders are useful for reducing the duration of catheter placement. ${ }^{26,48}$ Both of these approaches require appropriate, consistent documentation with input from bedside nurses, physicians, advanced practice providers, and information technology. Table 3 summarizes best practices for the prevention of CAUTI.

Importantly, CAUTI prevention supports broader antimicrobial stewardship. Over 55\% of inpatients receive at least one dose of an antibiotic during their hospital stay. ${ }^{48,49}$ In 2015, the White House released the National Action Plan for Combating Antibiotic-Resistant Bacteria with the goals of slowing the emergence of resistant bacteria, preventing the spread of antibiotic-resistant infections, and setting a target of a $20 \%$ reduction in the inappropriate use of antibiotics for hospitalized patients. ${ }^{50}$ Hospitalists care for a substantial number of 
TABLE 3. Best Practices for Prevention of CAUTI

1. Limit use of indwelling urinary catheters to only appropriate indications.

2. Remove indwelling urinary catheters as soon as possible, since the risk of CAUTI development increases with longer duration of use.

3. Consider the use of a condom or other external catheter or intermittent catheterization as alternatives to indwelling urinary catheters.

4. Use proper aseptic technique during catheter insertion.

5. Adhere to optimal catheter maintenance throughout duration of catheter use.

6. Collaborate with nurses and other healthcare providers in the development and implementation of catheter removal protocols.

Abbreviation: CAUTI, catheter-associated urinary tract infection

inpatients and, in turn, can drive actions to decrease CAUTIs and promote stewardship efforts. Through actions to decrease CAUTIs, hospitalists can promote these stewardship efforts.

\section{CONCLUSIONS}

CAUTI is one of the most common types of HAl and is associated with increased morbidity, hospital length of stay, and patient costs. Most CAUTIs are preventable by limiting the placement of unnecessary catheters to instances of true necessity and removing catheters when they are no longer clinically indicated. Proper technique for the insertion and maintenance of catheters is also important for reducing CAUTI rates. Hospitalists care for a substantial number of inpatients and can make major contributions to the appropriate diagnosis, treatment, and prevention of CAUTIs.

Disclosures: The authors have nothing to disclose.

\section{References}

1. Centers for Disease Control and Prevention. Estimates of healthcare-associated infections. Accessed September 27, 2018. http://www.cdc.gov/ncidod/ dhqp/hai.html

2. Calfee D. Crisis in hospital-acquired, healthcare-associated infections. Annu Rev Med. 2012;63(1):359-371. https://doi.org/10.1146/annurev-med-081210-144458

3. Scott R. The direct medical costs of healthcare-associated infections in US hospitals and the benefits of prevention. Centers for Disease Control and Prevention. 2009. Accessed Septemer 27, 2018. http://www.cdc.gov/ncidod/ dhap/pdf/Scott_CostPaper.pdf

4. Zimlachman E, Henderson D, Tamir O, et al. Health care associated infections. A meta-analysis of costs and financial impact on the US health care system. JAMA Intern Med. 2013;173(22):2039-2046. https://doi.org/10.1001/ jamainternmed.2013.9763

5. Nicolle LE. Catheter-acquired urinary tract infection: the once and future guidelines. Infect Control Hosp Epidemiol. 2010;31(4):327-329. https://doi. org/10.1086/651092

6. Weber DJ, Sickbert-Bennett EE, Gould CV, et al. Incidence of catheter-associated and noncatheter-associated urinary tract infections in a healthcare system. Infect Control Hosp Epidemiol. 2011;32(8):822-823. https://doi. org/10.1086/661107.

7. Healthcare-associated infections. National and state HAl progress reports or SIR reports. Accessed May 6, 2019. https://www.cdc.gov/hai/data/archive/ archive.html

8. Saint S. Clinical and economic consequences of nosocomial catheter-related bacteriuria. Am J Infect Control. 2000;28(1):68-75. https://doi.org/10.1016/ S0196-6553(00)90015-4

9. Platt R, Polk BF, Murdock B, Rosner B. Mortality associated with nosocomial urinary-tract infection. N Engl J Med. 1982;307(11):637-642. https://doi.
org/10.1056/NEJM198209093071101

10. Tambyah PA, Knasinski V, Maki DG. The direct costs of nosocomial catheter-associated urinary tract infection in the era of managed care. Infect Control Hosp Epidemiol. 2002;23(1):27-31.

11. Chant C, Smith OM, Marshall JC, Friedrich JO. Relationship of catheter-associated urinary tract infection to mortality and length of stay in critically ill patients: a systematic review and meta-analysis of observational studies. Crit Care Med. 2011;39(5):1167-1173. https://doi.org/10.1097/ CCM.0b013e31820a8581

12. Umscheid CA, Mitchell MD, Doshi JA, et al. Estimating the proportion of healthcare-associated infections that are reasonably preventable and the related mortality and costs. Infect Control Hosp Epidemiol. 2011;32(2):101-114. https://doi.org/10.1086/657912

13. Gould C, Umscheid C, Agarwal R, et al. Healthcare Infection Control Practices Advisory Committee (HICPAC): guideline for the prevention of catheter-associated urinary tract infections, 2009. Accessed November 1, 2018. http://www.cdc.gov/hicpac/pdf/CAUTI/CAUTIguideline2009final.pdf

14. Hooton TM, Bradley SF, Cardenas DD, et al. Diagnosis, prevention, and treatment of catheter-associated urinary tract infection in adults: 2009 International Clinical Practice Guidelines from the Infectious Diseases Society of America. Clin Infect Dis. 2010;50(5):625-663. https://doi.org/10.1086/650482.

15. Conway LJ, Larson EL. Guidelines to prevent catheter-associated urinary tract infection: 1980 to 2010. Heart Lung. 2011;41(3):271-283. https://doi. org/10.1016/j.hrtlng.2011.08.001

16. Greene L, Marx J, Oriola S. APIC elimination guide: guide to the elimination of catheter-associated urinary tract infections (CAUTIs). Accessed October 15, 2018. http://www.apic.org/Content/ NavigationMenu/PracticeGuidance/APICEliminationGuides/CAUTI_ Guide_0609.pdf

17. Yokoe DS, Mermel LA, Anderson DJ, et al. A compendium of strategies to prevent healthcare-associated infections in acute care hospitals. Infect Control Hosp Epidemiol. 2008;29(S1):S12-S21.

18. Wiley Z, Kobaidze K, Sexton ME, Jacob JT. Hospitalists as integral stakeholders in antimicrobial stewardship. Curr Treat Options Infect Dis. 2018;10(2):240248. https://doi.org/10.1007/s40506-018-0162-z

19. Claeys KC, Blanco N, Morgan DJ, Leekha S, Sullivan KV. Advances and challenges in the diagnosis and treatment of urinary tract infections: the need for diagnostic stewardship. Curr Infect Dis Rep. 2019;21(4):11. https://doi. org/10.1007/s11908-019-0668-7

20. Horan T, Andrus M, Dudeck M. CDC/NHSN surveillance definition of health care-associated infection and criteria for specific types of infections in the acute care setting. Am J Infect Control. 2008;36(5):309-332. https://doi. org/10.1016/j.ajic.2008.03.002

21. Nicolle LE, Gupta K, Bradley S, et al. Clinical practice guideline for the management of asymptomatic bacteriuria: 2019 update by the Infectious Diseases Society of America. Clin Infect Dis. 2019;68(10):e83-e110.

22. Donlan R. Biofilms and device-associated infections. Emerg Infect Dis. 2001;7(2):277-281. https://doi.org/10.3201/eid0702.010226

23. Choe HS, Son SW, Choi HA, et al. Analysis of the distribution of bacteria within urinary catheter biofilms using four different molecular techniques. Am J Infect Control. 2012;40(9):e249-e254. https://doi.org/10.1016/j.ajic.2012.05.010

24. Mohajer M, Darouiche R. Prevention and treatment of urinary catheter-associated infections. Curr Infect Dis Rep. 2013;15(2):116-123. https://doi. org/10.1007/s11908-013-0316-6

25. Weiner LM, Webb AK, Limbago B, et al. Antimicrobial-resistant pathogens 
associated with healthcare-associated infections: summary of data reported to the National Healthcare Safety Network at the Centers for Disease Control and Prevention, 2011-2014. Infect Control Hosp Epidemiol. 2016;37(11):12881301. https://doi.org/10.1017/ice.2016.174

26. Tambyah PA, Oon J. Catheter-associated urinary tract infection. Curr Opin Infect Dis. 2012;25(4):365-370. https://doi.org/10.1097/QCO.0b013e32835565cc

27. Warren JW, Tenney JH, Hoopes JM, Muncie HL, Anthony WC. A prospective microbiologic study of bacteriuria in patients with chronic indwelling urethral catheters. J Infect Dis. 1982;146(6):719-723. https://doi.org/10.1093/ infdis/146.6.719

28. Lo E, Nicolle L, Coffin S, et al. Strategies to prevent catheter-associated urinary tract infections in acute care hospitals: 2014 update. Infect Control Hosp Epidemiol. 2014;35(5):464-479. https://doi.org/10.1086/675718

29. Nicolle LE. Urinary catheter-associated infections. Infect Dis Clin N Am 2012;26(1):13-27. https://doi.org/10.1016/j.idc.2011.09.009

30. Titsworth WL, Hester J, Correia T, et al. Reduction of catheter-associated urinary tract infections among patients in a neurological intensive care unit: a single institution's success. J Neurosurg. 2012;116(4):911-920. https://doi. org/10.3171/2011.11.JNS11974

31. Lobdell KW, Stamou S, Sanchez JA. Hospital-acquired infections. Surg Clin N Am. 2012;92(1):65-77. https://doi.org/10.1016/j.suc.2011.11.003.

32. Gray M. Reducing catheter-associated urinary tract infection in the critical care unit. AACN Adv Crit Care. 2010;21(3):247-257.

33. Fakih MG, Watson SR, Greene T, et al. Reducing inappropriate urinary catheter use. Arch Intern Med. 2012;172(3):255-260. https://doi.org/10.1001/ archinternmed.2011.627

34. Saint S, Wiese J, Amory JK, Bernstein ML, et al. Are physicians aware of which of their patients have an indwelling urinary catheters? Am J Med. 2000;109(6):476-480. https://doi.org/10.1016/s0002-9343(00)00531-3

35. O'Grady NP, Barie PS, Bartlett JG, et al. Guidelines for evaluation of new fever in critically ill adult patients: 2008 update from the American College of Critical Care Medicine and the Infectious Diseases Society of America. Crit Care Med. 2008;36(4):1330-1349. https://doi.org/10.1097/CCM.0b013e318169eda9

36. Mullin K, Kovacs C, Fatica C, et al. A multifaceted approach to reduction of catheter-associated urinary tract infections in the intensive care unit with an emphasis on "stewardship of culturing." Infect Control Hosp Epidemiol. 2017;38(2):186-188. https://doi.org/10.1017/ice.2016.266

37. Humphries R, Bard J. Point-counterpoint: reflex cultures reduce laboratory workload and improve antimicrobial stewardship in patients suspected of having urinary tract infections. J Clin Microbiol. 2016;54(2):254-258. https:// doi.org/10.1128/JCM.03021-15

38. Voelker R. New fluoroquinolone warning. JAMA. 2016;315(23):2514. https:// doi.org/10.1001/jama.2016.7290
39. Tillotson GS. FDA and the safe and appropriate antibiotic use of fluoroquinolones. Lancet Infect Dis. 2016;16(3):E11-E12. https://doi.org/10.1016/ S1473-3099(16)00051-7

40. Tanne J. FDA adds "black box" warning label to fluoroquinolone antibiotics. BMJ 2008;337:a816. https://doi.org/10.1136/bmj.a816

41. Wyndaele JJ, Brauner A, Geerlings SE, et al. Clean intermittent catheterization and urinary tract infection: review and guide for future research. BJU Intern. 2012;110(11c):E910-E917. https://doi.org/10.1111/j.1464-410X.2012.11549.x

42. Hakwoort RA, Thijs SD, Bouwmeester FW, et al. Comparing clean intermittent catheterization and transurethral indwelling catheterization for incomplete voiding after vaginal prolapse surgery: a multicenter randomized trial. BJOG. 2011;118(9):1055-1060. https://doi.org/10.1111/j.1471-0528.2011.02935.x

43. Hakvoort RA, Nieuwkerk PT, Burger MP, et al. Patient preferences for clean intermittent catheterization and transurethral indwelling catheterization for treatment of abnormal post-void residual bladder volume after vaginal prolapsed surgery. BJOG. 2011;118(11):1324-1328. https://doi.org/10.1111 j.1471-0528.2011.03056.x

44. Saint $S$, Kaufman SR, Roger $M$, et al. Condom versus indwelling urinary catheters: a randomized trial. JAGS. 2006;54(7):1055-1061. https://doi. org/10.1111/j.1532-5415.2006.00785.x

45. Pickard R, Lam T, MacLennan G, et al. Antimicrobial catheters for reduction of symptomatic urinary tract infection in adults requiring short-term catheterization in hospital: a multicenter randomized controlled trial. Lancet. 2012;380(9857):1927-1935. https://doi.org/10.1016/S0140-6736(12)61380-4

46. Parry M, Grant B, Sestovic M. Successful reduction in catheter-associated urinary tract infections: focus on nurse-directed catheter removal. Am J Infect Control. 2013;41(12):1178-1181. https://doi.org/10.1016/j.ajic.2013.03.296

47. Fuchs MA, Sexton DJ, Thornlow DK, Champagne MT. Evaluation of an evidence-based, nurse-driven checklist to prevent hospital-acquired catheter-associated urinary tract infections in intensive care units. J Nurs Care Qual. 2011;26(2):101-109. https://doi.org/10.1097/NCQ.0b013e3181fb7847

48. Meddings J, Rogers MA, Macy M, Saint S. Systematic review and meta-analysis: reminder systems to reduce catheter-associated urinary tract infections and urinary catheter use in hospitalized patients. Clin Infect Dis. 2010;51(5):550-560. https://doi.org/10.1086/655133

49. Baggs J, Fridkin SK, Pollack LA, Srinivasan A, Jernigan JA. Estimating national trends in inpatient antibiotic use among US hospitals from 2006 to 2012. JAMA Intern Med. 2016;176(11):1639-1648. https://doi.org/10.1001/jamainternmed.2016.5651

50. National Action Plan for Combating Antibiotic-resistant Bacteria. 2015. Accessed April 28, 2019. https://obamawhitehouse.archives.gov/sites /default/files/docs/national_action_plan_for_combating_antibotic-resistant bacteria.pdf. 